November 27, 2018

\title{
High-Energy Unitarity and the Standard Model ${ }^{*}$
}

\author{
Alan. R. White ${ }^{\dagger}$ \\ Argonne National Laboratory \\ 9700 South Cass, Il 60439, USA.
}

\begin{abstract}
High-energy unitarity is argued to select a special version of QCD as the strong interaction. Electroweak symmetry breaking has to be due to a new sextet quark sector - that will produce large cross-section effects at the LHC. The sextet sector embeds, uniquely, in a massless $S U(5)$ theory that potentially generates the states and interactions of the Standard Model within a boundstate S-Matrix. Infra-red chirality transitions of the massless Dirac sea play an essential dynamical role.
\end{abstract}

Contributed to the Proceedings of the Gribov-75 Memorial Workshop.

*Work supported by the U.S. Department of Energy under Contract W-31-109-ENG-38

†arw@hep.anl.gov 


\section{Introduction.}

Vlodya Gribov devoted much of his life to studying the implications of unitarity for a high-energy S-Matrix. My own work is based heavily on Vlodya's ideas and formalisms and in this article, written in honor of what would have been his 75th birthday, I will outline results suggesting that high-energy unitarity could be a determining constraint on a particle S-Matrix. I will first argue that a special version of QCD $\left(\mathrm{QCD}_{S}\right)$, with experimentally attractive features, is selected as the strong interaction[1]. A new color sextet quark sector is present that has just the right properties to produce electroweak symmetry breaking - with large cross-section effects predicted for the LHC. A unique embedding[2] of the sextet sector in a left-handed $S U(5)$ theory $\left(\mathrm{GUT}_{S}\right)$ removes the sextet electroweak anomaly and also, amazingly, includes "almost exactly" the lepton and triplet quark sectors of the Standard Model, with $\mathrm{QCD}_{S}$ included in it's entirety. Because some of the $S U(2) \otimes U(1)$ quantum numbers are not quite right, $\mathrm{GUT}_{S}$ can not be a conventional unified theory. Rather, it may provide an underlying massless field theory which dynamically generates, within the bound-state S-Matrix, both the triplet and sextet hadronic sectors and, also, the leptonic sector of the Standard Model. If this proves ${ }^{\ddagger}$ to be the case then (it will be arguable that) unitarity uniquely determines the Standard Model !

My results imply that the infra-red chiral anomaly effects of a massless Dirac sea can have a deep significance in a bound-state S-Matrix. At high-energy, zero momentum chirality transitions (within reggeon vertices) produce states and amplitudes with dramatically different properties from those implied, at first sight, by the underlying field theory. In the S-Matrix of massless $\mathrm{QCD}_{S}$, the chirality transitions produce confinement and chiral symmetry breaking - with a minimal hadronic (triplet and sextet) spectrum[1], and Critical Pomeron high-energy behavior[3]. Although much remains to be understood about the S-Matrix of $\mathrm{GUT}_{S}$, it is already clear that, just because of the Dirac sea interactions, the Standard Model S-Matrix could indeed emerge. The $S U(5)$ symmetry is confined and so, very importantly, proton decay is not directly implied. In addition, because the chirality transitions occur only for vector coupled fermions, the real $S U(3) \otimes U(1)$ part of the theory dominates the $S$-Matrix. A resulting "wee gluon anomaly interaction" gives[1] a mass for SU(3) singlet left-handed vector bosons and the full interaction structure of the Standard Model appears. There is a related bound-state mass spectrum in which, because of the fermion representation structure, there are no (unwanted) symmetries.

I will emphasize where Gribov's work underlies the arguments that I present, beginning with the formulation of reggeon unitarity $[5,6]$ and ending with the importance of the dynamical role[7] of the Dirac sea. The Critical Pomeron solution[3]

\footnotetext{
${ }^{\ddagger}$ Most importantly, sextet electroweak symmetry breaking must be seen[1] at the LHC.
} 
of reggeon unitarity and the reggeization of non-abelian gauge theories (that has been established, in remarkable depth, by Lev Lipatov and collaborators[8]) are also crucial.

\section{Reggeon Unitarity}

The multi-regge behavior of scattering amplitudes is determined by partial-wave amplitudes analytically continued in complex angular momentum. The singularity structure in each "J-plane" is controlled by $t$-channel unitarity. Elastic unitarity generates regge poles which, in turn, generate regge cuts via multiparticle unitarity. The reggeon unitarity equations then determine the threshold discontinuity due to any combination of regge poles, in any partial-wave amplitude. If $\alpha(t)$ is a regge pole trajectory, the $M$-reggeon cut trajectory is $J=\alpha_{M}(t)=M \alpha\left(t / M^{2}\right)-M+1$.

When the reggeon unitarity equations were first derived[5], they were a spectacular intellectual jump and a profound reformulation and generalization of existing low-order diagrammatic calculations. There were, however, many uncertainties in the derivation that were resolved only after the development [6] of multiparticle symptotic dispersion relations as a fundamental basis for multi-regge theory. The generality of reggeon unitarity makes it extremely powerful, particularly when applied to the problem of constructing the multi-regge region QCD S-Matrix. First we discuss the abstract Reggeon Field Theory (RFT) solution of these equations.

\section{The Critical Pomeron}

The analogy with conventional unitarity makes it straightforward to formulate an effective field theory solution of reggeon unitarity. In fact, Gribov developed[9] a direct (underlying) diagrammatic formulation of RFT only because[6] of the uncertainties that, for a long time, surrounded the derivation of reggeon unitarity. We consider an, even-signature, pomeron regge pole with trajectory $j=\alpha(t)$. If $\alpha(0)=1$ then, also, $\alpha_{M}(0)=1$ and all the multipomeron singularities accumulate at one point. In this case, a simultaneous solution of all the discontinuity formulae is essential and all RFT diagrams have to be summed. Remarkably, a renormalization group formalism can be applied and a fixed-point solution shown to exist.

Under a renormalization group transformation, which rescales both $(1-J)$ and $k_{\perp}$, only the triple pomeron coupling $r$ survives as a relevant coupling. A fixed-point exists at $\alpha(0)=1$ and $r^{2}=\frac{4 \pi^{2}}{3} \epsilon+O\left(\epsilon^{2}\right)$ (where $\epsilon=4-D$ and $D$ is the dimension of $\left.k_{\perp}\right) . \epsilon=2$ gives an interacting pomeron theory with the "universality" property 
of a critical phenomenon. As a result, the asymptotic behavior can be precisely calculated without knowledge of the underlying "bare" parameters. Scaling laws can be derived for a wide variety of cross-sections and it can be shown that all known $s$ channel unitarity constraints on a theory of rising cross-sections are satisfied[10]. It can also be shown that, when $t$ is positive, all the multipomeron cuts separate and reggeon unitarity is satisfied. Therefore, the Critical Pomeron provides a complete, unique, solution of high-energy unitarity which, in a sense, represents the ultimate achievement of the pre-QCD program to construct a unique S-Matrix from unitarity. (It has been my goal, for a long time, to determinine how and when the Critical Pomeron occurs in a field theory.)

\section{The Supercritical Pomeron}

The supercritical phase[11] (obtained when, formally, $\alpha(0)>1$ ) plays a crucial role in linking the Critical Pomeron to an underlying field theory. Using a stationary point of the effective lagrangian introduces a "pomeron condensate" that generates new classes of RFT diagrams. Figure 1 contains a simple example of a new diagram. Reggeon unitarity determines that the $k_{\perp}$ poles produced by the two-pomeron prop-

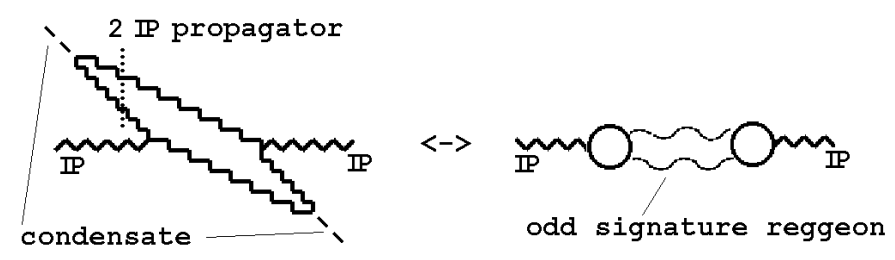

Figure 1: A new RFT diagram generated by the pomeron condensate

agators coupled to the condensate should be interpreted as particle poles lying on an odd-signature trajectory degenerate with that of the pomeron. The odd-signature reggeon couples pairwise to the pomeron and reggeon states involving many vector particle poles similarly appear in higher-order diagrams. In the supercritical phase, therefore, divergences in rapidity (produced by $\alpha(0)>1$ ) are converted to vector particle divergences in $k_{\perp}$ that are associated with the "deconfinement of a vector particle" on the pomeron trajectory.

\section{The Critical Pomeron and $\mathrm{QCD}_{S}$.}

If the Critical Pomeron is not present in a theory then, since perturbative crosssections rise, it is unlikely that large momentum perturbation theory can match 
smoothly with unitary forward amplitudes. In fact, before even discussing dynamical details, we can give some general arguments that specifically link the Critical Pomeron to a special version of QCD $\left(\mathrm{QCD}_{S}\right)$.

Breaking $S U(3)$ color to $S U(2)$ gives "color superconducting QCD" (CSQCD) in which a massive, reggeized, vector particle (a "massive gluon") is deconfined, just as is expected in the supercritical pomeron phase ${ }^{\S}$. However, at large momentum, a smooth transition from CSQCD to QCD is possible only if an asymptotically free scalar "Higgs" field can be employed. This is a strong requirement that is only possible $[1,13]$ if the asymptotic freedom constraint on QCD is "saturated" - immediately implying that there must be a further quark sector beyond that present in the conventional version of the Standard Model. Also, in the infra-red transverse momentum region, the transition from CSQCD to QCD can give the Critical Pomeron only if the infra-red divergences of perturbative reggeized gluon diagrams can generate non-perturbative pomeron diagrams describing a confining theory. As we will see, an infra-red fixed-point, that again exists only when asymptotic freedom is saturated, is crucial - in addition to chiral anomalies.

The saturation constraint (unrealistically) requires sixteen color triplet quarks or, alternatively, two color sextets and six triplets (giving $\mathrm{QCD}_{S}$ ). The resulting "sextet pions" provide, exactly, a "Higgs sector" producing electroweak mass generation $[1,14]$. Thus, requiring unitarity for the strong interaction leads directly to electroweak symmetry breaking and implies that there is a major sector of the strong interaction that has yet to be seen experimentally.

\section{$6 \mathrm{CSQCD}_{S}$ States and Amplitudes}

The derivation of supercritical pomeron diagrams required[11] the study of multiregge amplitudes in which the "pomeron condensate" could be understood as due to a "wee parton" component of scattering hadrons. Correspondingly, to derive supercritical states and amplitudes within $\mathrm{CSQCD}_{S}$, we have to consider a multiregge limit[15] in which the amplitude for the scattering of regge pole hadrons via pomeron exchange can emerge in it's entirety, as illustrated schematically in Figure 2. Fortunately, the generality of reggeon unitarity implies that the complicated reggeon diagrams involved can be constructed similarly to the well-known elastic scattering diagrams, the only difference being that vertices coupling distinct reggeon channels can contain anomalies not present in internal reggeon interactions.

The main infra-red divergence of massless gluon reggeon diagrams is due to

$\S$ That the gauge group has to be $S U(3)$ can be seen[12], without the detailed construction of the next Section, by using cut RFT to determine the center of the group. 


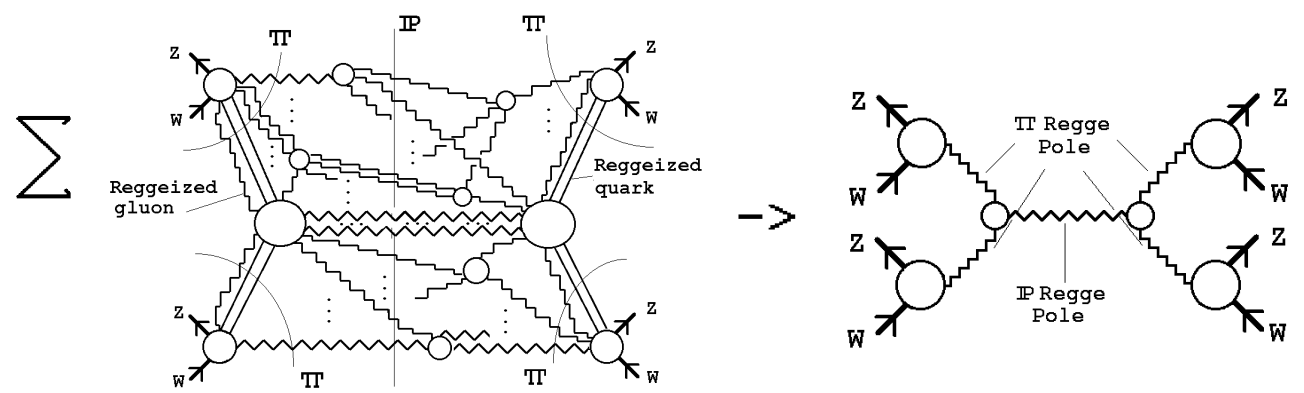

Figure 2: The transition from perturbative reggeon diagrams to reggeized pions scattering via pomeron exchange.

reggeization. This divergence exponentiates to zero all amplitudes with non-zero $S U(2)$ color in any reggeon channel. We consider diagrams, of the form shown in Figure 3, in which each reggeon state has two components, both of which carry zero
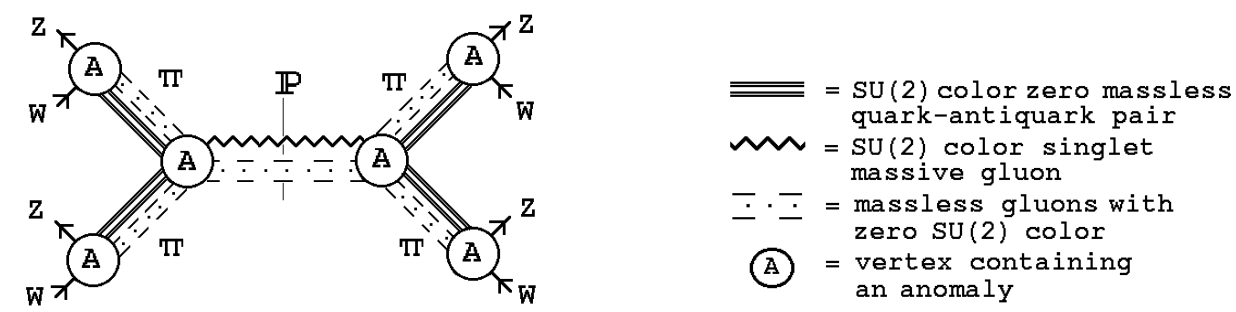

Figure 3: Diagrams with anomaly vertices that will produce the pion amplitude.

$S U(2)$ color. One component contains massless gluons while the other is either a quark-antiquark pair or a massive gluon reggeon. We choose external left-handed vector boson vertices because, as illustrated in Figure 4, they directly contain[16] a

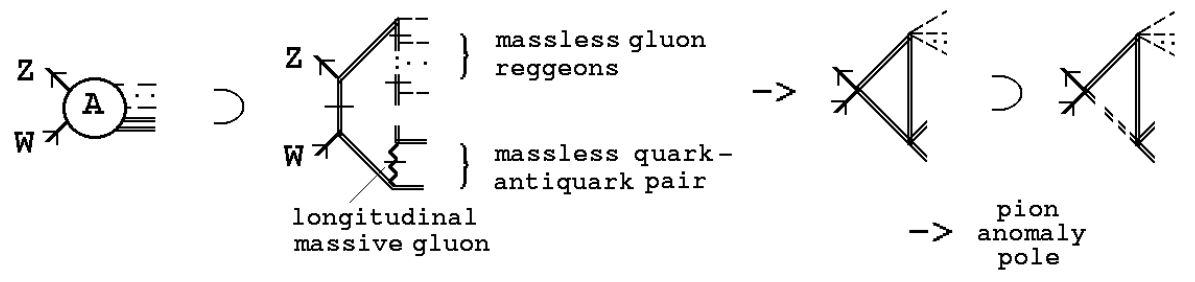

Figure 4: A reggeon vertex triangle diagram. The hatched lines are on-shell and the broken quark line indicates a zero momentum chirality transition.

triangle anomaly. Consequently, an "anomaly pole" appears, as shown, when the gluon reggeons carry zero transverse momentum. The internal vertices in Figure 3 contain U(1) anomaly diagrams of the form shown in Figure 5.

The initial effect of the anomalies is a large transverse momentum (nonunitary) power enhancement[16] of the high energy behavior. The enhancement can 


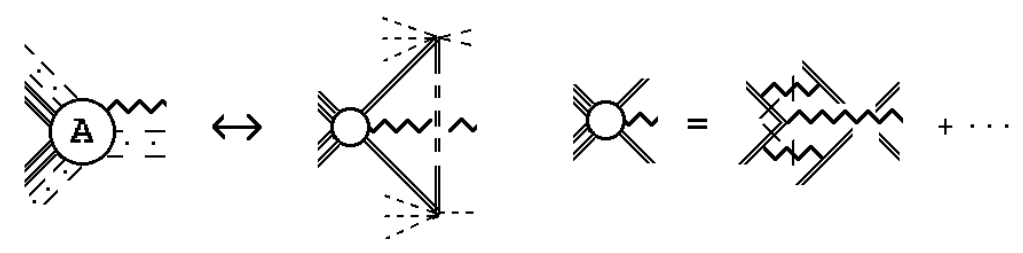

Figure 5: The reduction to a triangle diagram that involves the $\mathrm{U}(1)$ anomaly. The broken quark line again indicates the chirality transition giving the anomaly pole.

be removed with a transverse momentum cut-off but, because gauge invariance Ward identities are violated, a new infra-red divergence is produced. An overall (logarithmic) divergence is generated when all massless gluon transverse momenta are scaled uniformly to zero. (The $U(1)$ anomaly pole contributes as a transverse momentum conserving $\delta$-function.) However, when the massless gluon component in any reggeon state carries normal color parity (= signature), interactions with the second component in the state again exponentiate the divergence. For anomalous ( $\neq$ signature) color parity gluon components, these interactions are absent.

The infra-red fixed point, due to saturation, implies that color zero massless gluon reggeon interactions scale as all transverse momenta are scaled to zero. The overall "anomalous wee gluon" divergence is then preserved when interactions amongst the massless gluons are included. The residue of the divergence gives a physical $\mathrm{CSQCD}_{S}$ amplitude, in which the transverse momentum cut-off can be removed and all the massless gluons in Figure 3 contribute only as a "reggeon condensate". The condensate provides (crucial) zero transverse momentum, anomalous wee gluon, components in both the "pion" and the "pomeron".

$S U(2)$ anomalous gluons must have odd signature and so the $\mathrm{CSQCD}_{S}$ pomeron is an even signature regge pole which, because of the reggeon condensate, is exchange degenerate with a reggeized massive gluon, just as in supercritical RFT. Also because of the condensate, "pion" Goldstone boson anomaly poles", from the external vertices, appear in the physical amplitudes. By removing the transverse momentum cut-off after the extraction of anomaly infra-red divergences, we replace ultra-violet chirality violation (producing bad high-energy behavior) by infra-red chirality violation producing particle poles. This is how a confining, chiral symmetry breaking, bound-state spectrum is generated out of reggeon diagrams. The spectrum consists of triplet and sextet quark/antiquark "pions" and, due to the equivalence of conjugate $S U(2)$ representations, tripet and sextet quark/quark (and anti-quark/anti-quark) "nucleons".

IThis is related to the conformal invariance of Green's functions at the fixed-point.

"Via the chirality transition, the condensate is absorbed by a zero momentum anti-quark (or quark) that becomes unphysical, as in Gribov's confinement picture[7]. 


\section{Restoration of $S U(3)$ color}

There is every indication[1] that the reggeon diagrams of $C S Q C D_{S}$ map onto supercritical RFT, with the essential triple pomeron coupling given[17] by wee gluon anomaly interactions. It remains, however, a major challenge to carry out the mapping in detail. Assuming it can be done, the transition from $\operatorname{CSQCD}_{S}$ to $Q C D_{S}$ will necessarily give the Critical Pomeron. The reggeon condensate will disappear and the zero momentum chirality transitions (which can, equivalently, be regarded as Dirac sea shifts) in states and amplitudes will no longer be due to a semi-classical gauge field with fixed $S U(3)$ color. The transitions will remain and will be many in any scattering process, but they will correspond to random, dynamical, gauge field fluctuations within the color group. The transition from a fixed "magnetization" for the Dirac sea shifting gauge field, to a random, fluctuating, field characterizes the nature of the "critical phenomenon" that is associated with the high-energy behavior of $Q C D_{S}$. In effect, the longitudinal massive vector meson interactions do not decouple entirely as the color symmetry breaking is removed. They remain as dynamical fluctuations, but only at zero light-cone momentum. Including such interactions (specifically) amounts to fixing the (Gribov) ambiguity in the light-cone quantization of $\mathrm{QCD}_{S}$.

The quarks clearly have to be massless for the physics of $\mathrm{QCD}_{S}$ to be as we have described it. To add quark masses, and preserve the physics involved, appears non-trivial. The Dirac sea would have to undergo major shifts, of the kind envisaged by Gribov in his original confinement proposal[7], but in a random dynamical manner. In fact, the solution may be that effective quark masses are introduced via the boundstate masses originating from the embedding of $Q C D_{S}$ in $\mathrm{GUT}_{S}$, that we discuss below.

$\mathrm{QCD}_{S}$ baryons will be formed as bound states of $\mathrm{CSQCD}_{S}$ nucleons and $S U(2)$ singlet quarks. Crucially, because there are no chiral symmetries mixing the two sectors, there will be no "hybrid" sextet/anti-triplet/anti-triplet combinations. Therefore, the only new baryons will be the sextet proton and the (stable) sextet neutron.

\section{The Sextet QCD Scale and Electroweak Masses}

Sextet antiquarks have the same $S U(3)$ triality as triplet quarks and so we define "Standard Model" couplings for sextet antiquarks (quarks) to be the same as triplet quarks (antiquarks). In an infinite momentum hadron, wee gluons should reproduce vacuum properties and, indeed, an anomaly interaction generates a mass ${ }^{* *}$ for an exchanged vector boson, as illustrated in Figure 6. The sextet quark loop con-

\footnotetext{
** Similar interactions should also generate a pion mass in the even signature amplitude.
} 

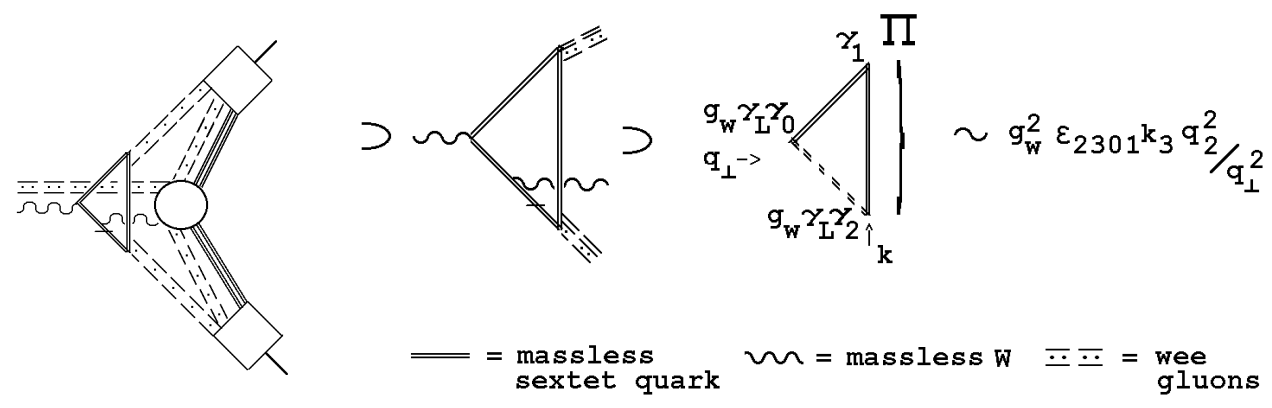

Figure 6: An Interaction Contributing to Vector Boson Mass Generation.

tribution is equivalent to the mixing ${ }^{\dagger \dagger}$ of a sextet pion (П) with a $W$ and gives $M_{W}^{2} \sim g_{W}^{2} \int d k k \equiv g_{W}^{2} F_{\Pi}^{2} \sim$ where $k$ is a wee gluon momentum. The wee gluon origin implies this mass appears only in the S-Matrix. It also occurs only for vectors with a left-handed coupling. No photon (or gluon) mass is generated !

Both sextet and triplet pions contribute to the vector boson mass generation but, sextet pions dominate because of larger color factors. Assuming the Casimir Scaling rule holds $\left(C_{6} \alpha_{s}\left(F_{\Pi}^{2}\right) \sim C_{3} \alpha_{s}\left(F_{\pi}^{2}\right)\right.$ with $C_{6} / C_{3} \approx 3$ ), if $\alpha_{s}$ evolves sufficiently slowly ( e.g. $\alpha_{s}\left(F_{\pi}^{2}\right) \sim 0.4$ ) then $F_{\Pi}$ can consistently be the electroweak scale! The large wee gluon coupling to sextet quarks also implies that the wee gluon component of the pomeron couples very strongly $\left(\sim F_{\Pi}\right)$ to sextet quarks. This strong coupling is a central element of our understanding of high-energy sextet crosssections.

\section{New Strong Interaction Physics}

In general, the larger color factors involved imply that the sextet sector will be a higher mass, stronger coupling, part of the strong interaction. As a result sextet states and cross-sections will dominate at high enough energy. At first sight, the proposition that the sought-after new physics "Beyond the Standard Model" is strong interaction physics seems very radical. However, it has a number of theoretical attractions and potentially answers a number of outstanding experimental problems, as the following list[1] illustrates.

1. Electroweak symmetry breaking involves no new interaction besides the $S U(3) \otimes$ $S U(2) \otimes U(1)$ gauge interactions of the Standard Model.

2. The electroweak scale is a new QCD (sextet chiral) scale, which Casimir scaling shows to be the right order of magnitude.

${ }^{\dagger \dagger}$ The anomaly chirality transition is necessary for this mixing. 
3. The spectrum is more limited than just color confinement and chiral symmetry breaking would imply, in better accord with experiment. Glueballs and quark resonances are excluded as asymptotic states. There is no BFKL pomeron and no odderon.

4. New large cross-section physics above the electroweak scale gives a natural explanation for many cosmic ray phenomena. Big increases in average transverse momenta, and in the production of sextet neutrals, could give the apparent "knee" in the spectrum.

5. The high-energy production of stable, neutral, sextet neutrons, in the early universe, provides a natural explanation for the dominance of dark matter formed as nuclei, clumps, etc. from sextet neutrons.

6. Being neutral and massive, sextet neutrons will avoid the GZK cut-off and so could be the mysterious, ultra-high energy, cosmic rays.

7. Top quark production could be due to the $\eta_{6}$ and sextet quark effects could explain an excess, large $E_{T}$, jet cross-section at the Tevatron.

\section{LHC Physics}

If the cosmic ray spectrum knee is associated with an effective threshold for sextet quark physics (the energy is right, given that pomeron production is needed), large cross-section effects must appear very rapidly with energy to make the knee visible. Such effects should be apparent at the LHC, with dramatic new physics involved[1]. Considering anomaly pole amplitudes, we can show that the strong coupling of the pomeron to sextet states implies that hard double pomeron production of electroweak vector bosons will give jet cross-sections comparable with normal QCD jet (nondiffractive) cross-sections. In particular, the boson pair cross-section is estimated to be, roughly, twelve orders of magnitude larger than in the Standard model. Combining this estimate with pomeron regge theory gives a small transverse momentum cross-section that is correspondingly large. During the initial "soft physics" running period, it should be straightforward[18] to look for the vector boson pairs in the CMS central detector that should be produced in combination with scattered protons in the TOTEM Roman Pots.

If pomeron exchange amplitudes are large, then cut-pomeron amplitudes should also be large. As a result, we expect very large inclusive cross-sections for sextet states (multiple vector bosons, in particular) across most of the rapidity axis. This implies that jet cross-sections, at very large transverse momentum, will be orders of magnitude larger than expected. The production cross-section for sextet nucleon pairs should also be hadronic in size, although stable sextet neutrons (dark matter!) may 
be difficult to detect. If the sextet nucleon double pomeron cross-section is extraordinarily large, it might be detectable in the low luminosity run. If not, it might be seen by the high luminosity detectors[18] that will look for double pomeron production of the Standard Model Higgs particle.

\section{$11 \mathrm{GUT}_{S}$}

Well above the electroweak scale, the $Q C D_{S}$ infra-red fixed point requires $\alpha_{s} \lesssim \frac{1}{34} \sim$ $\alpha_{e w}$, implying that the sextet sector can, naturally, produce the decrease in $\alpha_{s}$ needed for unification. (Supersymmetry is not required !) A priori, unification could also determine how the $S U(2) \otimes U(1)$ sextet sector anomaly is canceled, as well as providing an origin for masses.

Many years ago (with Kyungsik Kang) we found[2] a remarkable, but puzzling, result. We looked at asymptotically free, anomaly-free. left-handed unified theories that contain the sextet sector, We discovered that a unique theory is selected, i.e. $S U(5)$ gauge theory with the fermion representation $5+15+40+45^{*}\left(\equiv \mathrm{GUT}_{S}\right)$. (In any higher unitary group the sextet sector requires a representation that is too large for asymptotic freedom.) Under $S U(3) \otimes S U(2) \otimes U(1)$

$$
\begin{aligned}
& 5=\left(1,3,-\frac{1}{3}\right)+\left(2,1, \frac{1}{2}\right), \quad 15=(1,3,1)+\left(3,2, \frac{1}{6}\right)+\left\{\left(6,1,-\frac{2}{3}\right)\right\}, \\
& 40=\left(1,2,-\frac{3}{2}\right)+\left(3,2, \frac{1}{6}\right)+\left(3^{*}, 1,-\frac{2}{3}\right)+\left(3^{*}, 3,-\frac{2}{3}\right)+\left\{\left(6^{*}, 2, \frac{1}{6}\right)\right\}+(8,1,1), \\
& 45^{*}=\left(1,2,-\frac{1}{2}\right)+\left(3^{*}, 1, \frac{1}{3}\right)+\left(3^{*}, 3, \frac{1}{3}\right)+\left(3,1,-\frac{4}{3}\right)+\left(3,2, \frac{7}{6}\right)+\left\{\left(6,1, \frac{1}{3}\right)\right\}+\left(8,2,-\frac{1}{2}\right)
\end{aligned}
$$

The triplet quark and lepton sectors, although not asked for, are remarkably close to the Standard Model. There are three "generations" of quarks/anti-quarks, with quark charges $\frac{2}{3}$ and $-\frac{1}{3}$, and three "generations" of $S U(2)$ doublet $(S U(3)$ singlet) leptons. The puzzle is that the $S U(2) \otimes U(1)$ quantum numbers are almost, but not quite, right and there are also (apparently unwanted) color octet quarks with lepton-like electroweak quantum numbers. At the time[2], we considered various "anomalous fermion phenomena", but found no convincing dynamical route to the Standard Model.

\section{A Massless Theory of Matter?}

In fact, $\mathrm{GUT}_{S}$ has similar properties to massless $Q C D_{S}$. Asymptotic freedom is saturated and an infra-red fixed-point keeps the $S U(5)$ coupling very small. Also, the $S U(5)$ symmetry can be broken to $S U(4)$ with an asymptotically free scalar field and so the high-energy S-Matrix can be constructed via reggeon diagram anomaly 
interactions introduced by the symmetry breaking. Because infra-red divergences will confine $S U(5)$ color in the S-Matrix, all elementary fermions will be massless and confined. The massless Dirac sea will control the dynamics, but with a crucial difference from $\mathrm{QCD}_{S}$. In $\mathrm{GUT}_{S}$, left-handed fermion interactions will exponentiate the anomalous color parity divergences that produce the states and amplitudes of $\mathrm{QCD}_{S}$. Therefore, these divergences will only be produced by the $S U(3) \otimes U(1)$ vector part of the theory. The left-handed vector bosons, with no SU(3) color, will aquire a mass, as in Section 8. Thus, just the interactions of the Standard Model will be selected within the $\mathrm{GUT}_{S}$ S-Matrix.

As yet, very little is certain, but our current ideas[4] about the S-Matrix construction, and other properties of $\mathrm{GUT}_{S}$, can be listed as follows. (We use $\left.S U(5) \rightarrow S U(3)_{C} \otimes S U(2)_{L} \otimes U(1) \rightarrow S U(2)_{C} \otimes S U(2)_{L} \otimes U(1).\right)$

- We begin with a $k_{\perp}$ cut-off and $S U(5)$ broken to $S U(2)_{C}$.

- The states are $S U(2)_{C}$ singlet Goldstones ( $\pi$ 's ) which are $q \bar{q}$ pairs in an $S U(2)_{C}$ condensate, as in $C S Q C D_{S}$.

- The $\pi$ 's contain q's that are 3's, 6's, and 8's under $S U(3)_{C}$. (8's are real wrt $S U(3)_{C}$, but give complex doublets wrt $S U(2)_{C} \cdot$ )

- Anomaly interactions, involving the $S U(2)_{C}$ condensate, generate $W^{ \pm}$and $Z^{0}$ masses via mixing (predominantly) with sextet $\pi$ 's.

- Similar interactions should generate $\pi$ masses. The pattern, and how many parameters are involved, remains to be studied.

- Restoring $S U(2)_{L}$ symmetry gives $S U(2)_{C} \times S U(2)_{L}$ invariant states.

- "Octet pions" form bound state leptons with elementary "leptons".

- After $S U(2)_{L}$ confinement, the right-handed sextet flavor dependence gives a (broken) $S U$ (2) S-Matrix symmetry.

- With $S U(3)_{C}$ color, the Pomeron is Critical and the photon massless.

- SU(3) octet quarks have no chiral anomaly and hence no pomeron coupling hence no strong interaction! They appear in bound-state leptons as pairs of positive and negative energy massive states.

- Because the coupling is so small, the underlying SU(5) theory will be close to conformal and the smallest lepton masses will be very small.

- There are no spectrum symmetries that would prevent the S-matrix from having the full structure of the Standard Model.

- The (experimentally attractive) SU(5) value of the Weinberg angle should be valid for $G U T_{S}$, even though there is no proton decay!

- The 144 representation of $S O(10)$ is anomaly free, asymptotically free, and contains $G U T_{S}$. There is, however, no "saturation" and so we can not construct a unitary, anomaly based, high-energy S-Matrix. 


\section{Comments}

The success of the Standard Model is a major scientific achievement. Nevertheless, it remains an enigma in the sense that there are a large number of parameters and there is no understanding of why it has been chosen by nature. The candidate theories/ideas that have so far been proposed for extending the model (including supersymmetry and superstrings) have failed to provide any understanding of the model's uniqueness and, as a result, have provided a wide range of possible extensions.

In the search to understand the origin of the Standard Model, the importance of asking that physical states give a unitary S-Matrix may been underestimated. When S-Matrix theory reigned supreme[6], before the dramatic rebirth of field theory that has led to the Standard Model, awareness of the difficulty involved led to the proposal that there is a unique S-Matrix that could, in principle, be bootstrapped from unitarity.

In an asymptotically free field theory, the wild divergence (non-summability) of perturbative interactions occurs in the infra-red region. Presumably, it is very difficult to keep this divergence out of the S-Matrix. Perhaps, there has to be a drastic reduction of the field theory degrees of freedom, in the states and in the interactions, that is only achievable (in very special circumstances) via infra-red anomalies of the massless Dirac sea. Could it be that the unitary S-Matrix is indeed unique but that to construct it, knowledge of the (also unique) underlying massless field theory is essential?

\section{References}

[1] A. R. White, "The Physics of a Sextet Quark Sector", hep-ph/0412062, to be published in Physical Review D.

[2] K. Kang and A.R. White, Int. J. Mod. Phys. A2, 409 (1987).

[3] A. A. Migdal, A. M. Polyakov and K. A. Ter-Martirosyan, Zh. Eksp. Teor. Fiz. 67, 848 (1975); H. D. I. Abarbanel and J. B. Bronzan, Phys. Rev. D9, 2397 (1974)

[4] A. R. White, "A Unique Massless Theory of Matter?", to appear.

[5] V. N. Gribov, I. Ya. Pomeranchuk and K. A. Ter-Martirosyan, Phys. Rev. 139B, 184 (1965).

[6] A. R. White, "The Past and Future of S-Matrix Theory" in "Scattering" eds. E. R. Pike and P. Sabatier (Academic Press, 2002), and references therein. 
[7] For details of Gribov's confinement picture and for references to the original papers see Y. L. Dokshitzer, presentation at this conference (2005).

[8] V. S. Fadin, E. A. Kuraev and L. N. Lipatov, Sov. Phys. JETP 45, 199 (1977); V. S. Fadin and L. N. Lipatov, Nucl. Phys. B406, Nucl. Phys. B477, 767 (1996) and further references therein; J. Bartels, V. S. Fadin and R. Fiore, Nucl. Phys. B672, 329 (2003) and references therein.

[9] V. N. Gribov, Soviet Phys. JETP 26, 414 (1968).

[10] M. Moshe, Phys. Rept. 37, 255 (1978).

[11] A. R. White, Int. J. Mod. Phys. A6, 1859 (1991).

[12] A. R. White, "The Pomeron in QCD", CERN-TH-2976 (1980).

[13] D. J. Gross and F. Wilczek, Phys. Rev. D8, 3633 (1973).

[14] W. J. Marciano, Phys. Rev. D21, 2425 (1980); see also E. Braaten, A. R. White and C. R. Willcox, Int. J. Mod. Phys., A1, 693 (1986).

[15] A. R. White, Phys. Rev. D58, 074008 (1998) describes the relevant multi-regge limit and the reggeon unitarity construction of multi-reggeon diagrams.

[16] A. R. White,Phys. Rev. D69, 096002 (2004).

[17] A. R. White,Phys. Rev. D66, 045009 (2002).

[18] M. G. Albrow, hep-ex/0507095, hep-ex/0409304. 